\title{
Dichloroacetic acid up-regulates hepatic glutathione synthesis via the induction of glutamate-cysteine ligase
}

\author{
Angelo Theodoratos ${ }^{a}$, Anneke C. Blackburn ${ }^{a}$, Jean Cappello ${ }^{a}$, Padmaja Tummala ${ }^{a}$, \\ Jane E. Dahlstrom ${ }^{\mathrm{a}, \mathrm{b}}$, Philip G. Board ${ }^{\mathrm{a}, *}$ \\ a John Curtin School of Medical Research, Australian National University, PO Box 334, Canberra, ACT 2601, Australia \\ ${ }^{\mathrm{b}}$ Anatomical Pathology, ACT Pathology, The Canberra Hospital, Garran, ACT, Australia
}

\section{A R T I C L E I N F O}

\section{Article history:}

Received 13 October 2011

Accepted 15 November 2011

Available online 23 November 2011

\section{Keywords:}

Acetaminophen toxicity

Dichloroacetic acid

Glutathione

Glutamate-cysteine ligase

Liver

Pathology

\begin{abstract}
A B S T R A C T
Dichloroacetic acid (DCA) has potential for use in cancer therapy and the treatment of metabolic acidosis. However, DCA can create a deficiency of glutathione transferase Zeta (GSTZ1-1). Gstz1 knockout mice have elevated oxidative stress and low glutathione levels that increases their sensitivity to acetaminophen toxicity. As it is highly likely that patients that are treated with DCA will develop drug induced GSTZ1-1 deficiency we considered they could be at risk of elevated toxicity if they are exposed to other drugs that cause oxidative stress or consume glutathione (GSH). To test this hypothesis we treated mice with DCA and acetaminophen (APAP). Surprisingly, the mice pre-treated with DCA suffered less APAP-mediated hepatotoxicity than untreated mice. This protection is most likely due to an increased capacity for the liver to synthesize GSH, since DCA increased the expression and activity of glutamate-cysteine ligase GCL, the rate-limiting enzyme of GSH synthesis. Other pathways for acetaminophen disposal were unchanged or diminished by DCA. Pre-treatment with DCA may be of use in other settings where the maintenance of protective levels of GSH are required. However, DCA may lower the efficacy of drugs that rely on oxidative stress and the depletion of GSH to enhance their cytotoxicity or of drugs that are detoxified by GSH conjugation. Consequently, as the use of DCA in the clinic is likely to increase, it will be critical to evaluate the interactions of DCA with other drugs to ensure the combinations retain their efficacy and do not cause enhanced toxicity.
\end{abstract}

(c) 2011 Elsevier Inc. All rights reserved.

\section{Introduction}

There is an increasing interest in the therapeutic use of dichloroacetic acid (DCA) in the treatment of cancer and lactic acidosis [1-4]. The use of DCA in the treatment of lactic acidosis and as an anti-cancer agent results from its capacity to inhibit pyruvate dehydrogenase kinase that inturn regulates pyruvate dehydrogenase and subsequently the metabolism of lactate and/or pyruvate through the citric acid cycle. Many tumor cells have a glycolytic phenotype and derive most of their ATP from glycolysis. DCA can reverse the glycolytic phenotype, depolarise the hyperpolarized inner mitochondrial membrane to normal levels and increase mitochondrial metabolism. It has been demonstrated that these effects can inhibit the growth of metastatic breast cancer, pancreatic cancer and glioblastoma in vivo [1-3,5]. Previous clinical trials in mitochondrial disorders have indicated that DCA has low toxicity and can be taken orally [4,6]. Several clinical trials of DCA against cancer are underway, including for

\footnotetext{
* Corresponding author. Tel.: +612 6125 4714; fax: +6126125 4712 .

E-mail address: Philip.Board@anu.edu.au (P.G. Board).
}

brain cancer [2], metastatic solid cancers, metastatic breast cancer and head and neck cancers, often combining DCA treatment with current chemotherapy or radiation therapy approaches (clinicaltrials.gov). Although clinical trials demonstrating the efficacy of DCA in cancer therapy have not been completed, the wide spread publicity of Bonnet et al.'s report [1] together with the ready access and oral availability of DCA has created patient demand for DCA treatment, and in some cases extensive patient self medication [7].

DCA is a substrate for glutathione transferase Zeta (GSTZ1-1) but can also act as a mechanism-based inactivator. Consequently, the treatment of mice with DCA creates a severe deficiency of GSTZ1-1 [8-10] and it is likely that DCA therapy in humans could also cause a significant GSTZ1-1 deficiency. Because of the role of GSTZ1-1 in tyrosine catabolism (GSTZ1 catalyses the isomerisation of maleylacetoacetate to fumarylacetoacetate [11]), Gstz1-deficient mice accumulate electrophilic intermediates such as maleylacetoacetate and are subject to constitutive oxidative stress resulting in chronically low hepatic GSH levels [12].

GSH is vitally important for the maintenance of intracellular redox balance and the disposition of a range of therapeutic drugs and other xenobiotics [13]. Low GSH levels can result in adverse chemical toxicity. It is highly likely that patients who are treated 
with DCA or patients who self-medicate with DCA will also be exposed to a range of other compounds that may require GSH for their function or disposition. Acetaminophen (APAP) is a prime example as it is widely available and causes hepatotoxicity when GSH is depleted. We considered that patients taking APAP or other GSH-consuming drugs could be at risk of hepatotoxicity if the doses of DCA are high enough to inactivate most of the GSTZ1-1 and recapitulate the oxidative stress and low GSH levels that occur in Gstz1-deficient mice. To evaluate this possibility, we treated mice with DCA to inactivate GSTZ1-1, and then evaluated hepatic GSH levels and markers of oxidative stress. Surprisingly, the mice treated with DCA exhibited an enhanced capacity for GSH synthesis as a consequence of elevated levels of the catalytic subunit of glutamate-cysteine ligase (GCLC). When subsequently treated with APAP the DCA-treated mice suffered less apparent hepatotoxicity than untreated mice. The capacity of DCA to replenish GSH levels in response to high levels of GSH consumption is an unexpected discovery that has implications for the use of DCA in conjunction with other therapies.

\section{Materials and methods}

\subsection{Reagents}

All chemicals were obtained from Sigma unless otherwise noted.

\subsection{Animals}

All mice used in this study were of the BALB/c strain and all treatment was approved by the Institutional Animal Experimentation Ethics Committee and were consistent with the NHMRC code of practice. Male mice, 10-15 weeks old were injected intraperitoneally (i.p.) with DCA (pH 7.2) at a daily rate of $250 \mathrm{mg} /$ $\mathrm{kg}$ diluted in normal saline. APAP was given as a single i.p. injection of $300 \mathrm{mg} / \mathrm{kg}$ diluted in normal saline.

\subsection{Western blotting and antiserum}

Livers were homogenised in T-PER lysis buffer (Thermo Scientific, Rockford, IL, USA). Proteins were quantitated with the BCA protein assay kit (Thermo Scientific, Rockford, IL, USA) and $50 \mu \mathrm{g}$ of total liver proteins were resolved per lane by SDSPAGE, transferred to PVDF membrane and probed with the following: Anti-GSTZ1 and anti-glutathione synthetase (GS) antisera (prepared in our laboratory from rabbits with recombinant human GSTZ1-1 and recombinant human GS, respectively), anti-glutamate-cysteine ligase modifier subunit (GCLM), antiNADPH quinone oxioreductase (NQO1) and $\beta$-actin antibodies (Abcam, Cambridge, MA, USA), and anti-glutamate-cysteine ligase catalytic subunit (GCLC) antibody (purchased from Novus Biologicals, Littleton, CO, USA). Proteins were detected by enhanced chemiluminescence reagents (GE Healthcare, NSW, Australia).

\subsection{Glutathione and enzyme assays}

The concentrations of glutathione in mouse livers was determined by a glutathione reductase recycling assay [14] as previously described [12]. The level of plasma alanine aminotransferase (ALT) was determined spectrophotometrically by linking the reaction to the oxidation of NADH to NAD. The activity of glutamate-cysteine ligase was determined in liver extracts by an assay that detects $\gamma$-glutamylcysteine after derivatization with 2,3-napthalenedicarboxaldehyde (Aldrich Castle Hill, NSW, Australia) [15].

\subsection{Real time RT-PCR}

RNA was extracted from liver tissue using a Qiagen RNeasy kit (QIAGEN Doncaster VIC Australia) and reverse transcribed into cDNA using Superscript III reverse transcriptase (Invitrogen, VIC, Australia). Real time PCR was performed with Power SYBR green reagents (Invitrogen, VIC, Australia) and run on an ABI thermal cycler (Applied Biosystems, VIC, Australia). Expression levels were normalised to ubiquitin-conjugating enzyme E2D2 (UBE2D2) expression.

\subsection{UDP-glucuronidation}

UDP glucuronyltransferase (UGT) activity was assayed in liver microsomes with a UDP GLO Assay kit according to the manufacturer's instructions (Promega, WI, USA). Briefly, a UGT substrate (lumiscent when glucuronic acid is unconjugated) was incubated with liver lysates in the prescence of uridine 5'diphosphoglucuronic acid. The loss of luminescence, was proportional to the UGT activity and was measured in a Glomax 96 microplate luminometer (Promega, WI, USA).

\subsection{Histopathology}

Mice were sacrificed by cervical dislocation and livers were fixed immediately in 10\% neutral phosphate-buffered formalin before processing and embedding into paraffin using standard laboratory methods. Sections were cut from each sample $(4 \mu \mathrm{m})$ and stained with hematoxylin and eosin.

The following criteria were applied in grading the severity of the hepatic congestion, necrosis and steatosis modified from Blazka et al. [16].

Grade 0: normal histology.

Grade 1: characterized by minimal congestion and necrosis of single hepatocytes (balloon degeneration), limited to the area immediately around the centrilobular vein (zone 3); many of the lobules not affected. Mild macrovesicular steatosis (fatty change) with occasional groups of neutrophils [mild steatohepatitis].

Grade 2: characterized by moderate congestion and haemorrhage of the area around the centrilobular vein (zone 3 ) and extending into the midzonal cells (zone 2); most lobules are affected. Areas of confluent necrosis limited to the liver cells surrounding the centrilobular vein. Moderate macrovesicular steatosis with occasional groups of neutrophils [moderate steatohepatitis].

Grade 3: characterized by widespread areas of congestion and haemorrhage in the centrilobular (zone 3 ) and midzonal (zone 2 ) areas of the liver. Confluent coagulative necrosis involving all hepatocytes in the centrilobular zone; bridging of areas of necrosis between centrilobular zones is common. Moderate macrovesicular steatosis with multiple groups of neutrophils [severe steatohepatitis].

\section{Results}

We have previously shown that treatment of male BALB/c mice with a single daily intraperitoneal injection of $250 \mathrm{mg} / \mathrm{kg}$ of DCA for 5 days causes a significant decrease in the level of hepatic GSTZ1-1 [17]. This dosing regimen was used in this study to create a chemical knockout of GSTZ1-1. Genetic Gstz1 knockout mice suffer from constitutive oxidative stress that results in the strong induction of NADPH quinone oxidoreductase (NQO1) and other enzymes that are regulated by the Keap1/Nrf2 pathway [12]. NQO1 
expression in the liver of mice treated with DCA was examined by Western blotting (Fig. 1). There was at best a marginal increase in NQO1 expression after DCA treatment that was far less than the level of NQO1 expression in genetic Gstz1 knock out mice. This indicates that the depletion of GSTZ1-1 induced by the DCA dosing regimen used is insufficient to generate the level of oxidative stress that occurs in genetic GSTZ1-1 deficiency. However, we noted that the levels of the catalytic subunit of glutamate-cysteine ligase (GCLC) were elevated but the levels of the modulating subunit of glutamate-cysteine ligase (GCLM), and glutathione synthetase were unchanged (Fig. 1). Consistent with this observation the activity of glutamat-cysteine ligase was found to be significantly elevated in mice treated with DCA (Fig. 2a). Glutamate-cysteine ligase is considered to be the rate-limiting step in glutathione synthesis and high activity would be conducive to an increased capacity for glutathione synthesis [18]. The level of glutathione in the liver of DCA treated mice is only slightly higher than in untreated mice (Fig. 2b) but is strikingly higher than the low levels of GSH $(\approx 2 \mu \mathrm{mol} / \mathrm{g}$ ) we have previously noted in the liver of Gstz1 knock out mice [11]. The minimal NQO1 induction and the normal GSH levels suggest that the DCA-mediated induction of GCLC is independent of the oxidative-stress mediated-effects seen in genetically determined GSTZ1-1 deficiency.

\subsection{DCA up regulates GCLC, prevents GSH depletion and reduces acetaminophen hepatotoxicity}

As a model of the potential impact of DCA on drug therapy, we evaluated the effect of DCA on APAP hepatotoxicity. APAP hepatotoxicity occurs after cellular GSH is completely consumed by its conjugation to N-acetyl-p-benzoquinone iminine (NAPQI), a toxic intermediate produced from APAP $[19,20]$. Where APAP administration resulted in complete depletion of hepatic glutathione in control mice $3 \mathrm{~h}$ after the APAP dose, GSH levels remained at near-normal levels in mice that had been pre-treated with DCA (Fig. 3a). GSH levels had recovered by $6 \mathrm{~h}$ post-APAP administration in all treatment groups, as has been observed elsewhere [21].

The release of ALT into the circulation is a measure of hepatic toxicity and we found that it was significantly elevated in control mice 3 and $6 \mathrm{~h}$ after receiving APAP (Fig. 3b). Treatment of mice

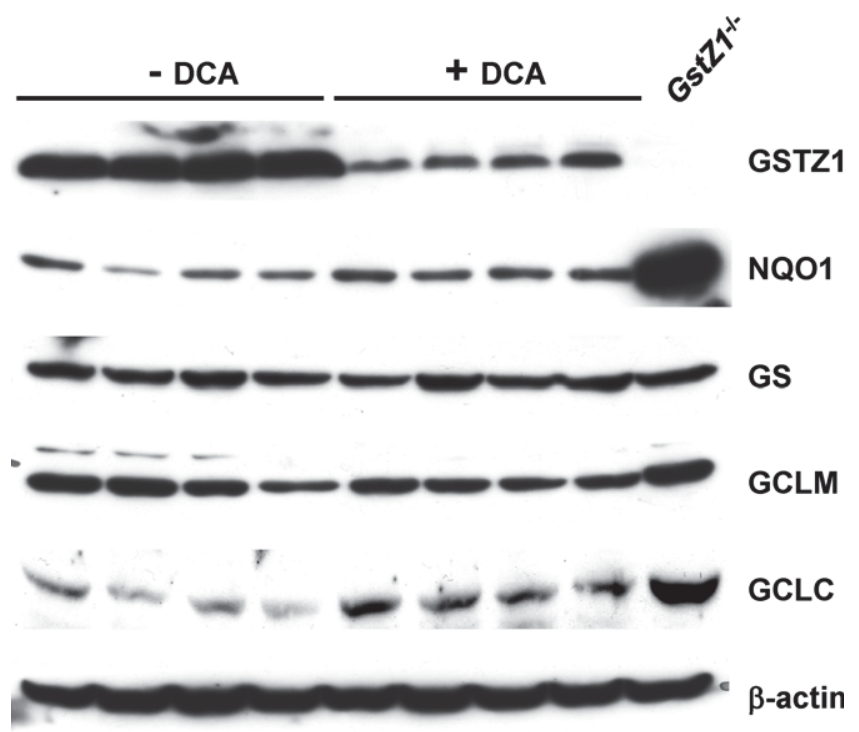

Fig. 1. DCA attenuates GSTZ1 and enhances GCLC protein levels. Male BALB/c mice $(10-15$ weeks old) were untreated $(n=6)$ or treated $(n=6)$ daily with $250 \mathrm{mg} / \mathrm{kg}$ DCA i.p. for 5 days. Liver cytosolic proteins were resolved and probed with the indicated antibodies. Samples from four different mice in each group are shown as well as a BALB/c Gstz1 $1^{-1-}$ control mouse. with DCA for 5 days prior to treatment with APAP prevented the release of ALT into plasma that occurred in APAP-only treated mice.

Histological studies of the liver also confirmed that prior treatment with DCA protected mice against the hepatotoxic effects of APAP (Fig. 4). Light microscopy of the livers from the control mice showed no histological abnormalities in the presence or absence of DCA (Grade 0) (Fig. 4a). However, characteristic changes due to NAPQI toxicity $[16,19]$ were evident $3 \mathrm{~h}$ or $6 \mathrm{~h}$ after the administration of APAP (Grade $1-2$ at $3 \mathrm{~h}$ and Grade $2-3$ at $6 \mathrm{~h}$ ) (Fig. 4b). The administration of DCA for five days prior to APAP administration totally prevented the acute liver injury (Fig. 4c).

Light microscopy of the livers from the $G s t z 1^{-1-}$ mice treated with APAP showed that the administration of APAP for $3 \mathrm{~h}$ resulted
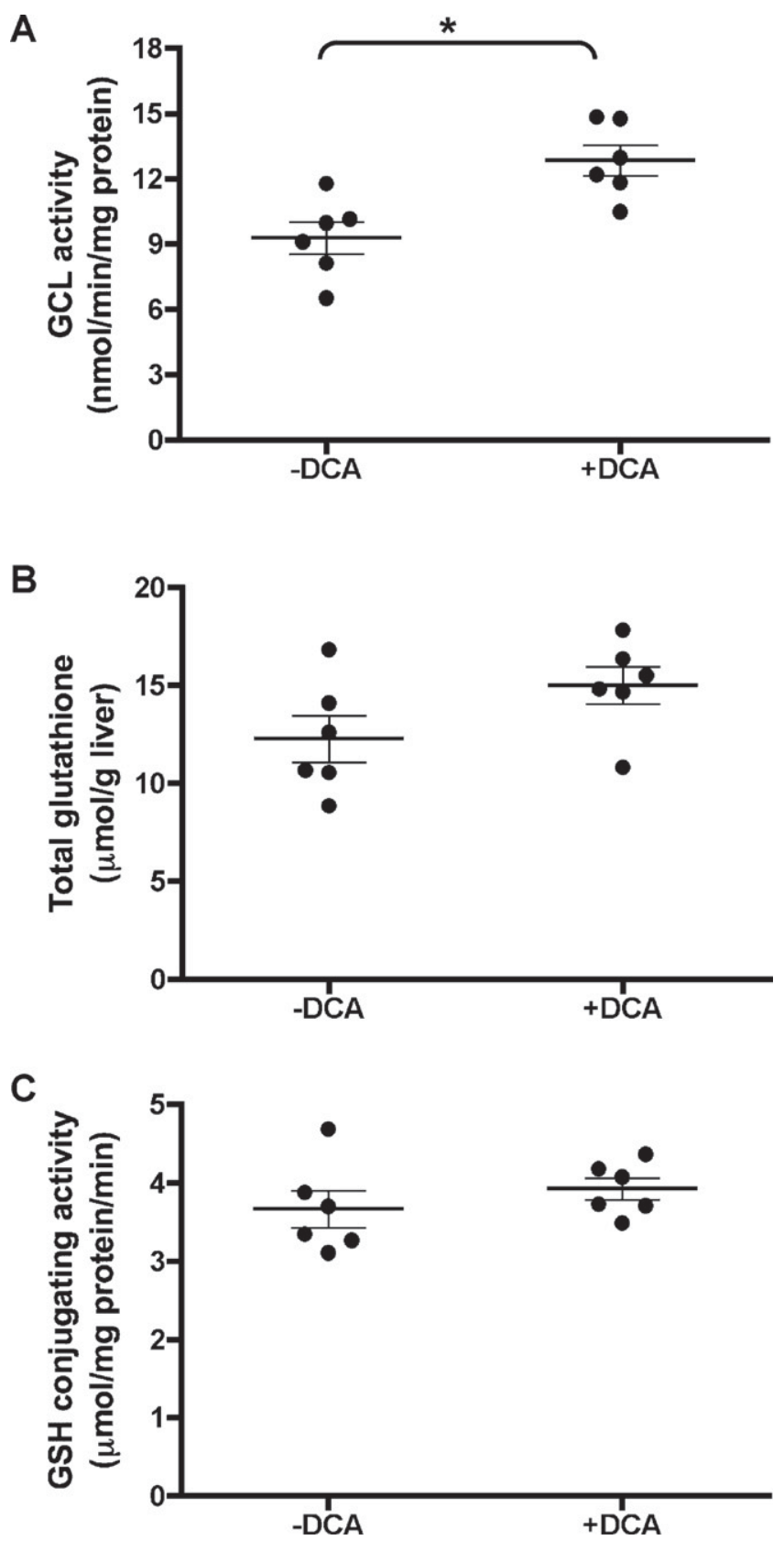

Fig. 2. The effect of DCA on glutamate-cysteine ligase and glutathione transferase activity. Groups of male BALB/c mice (10-15 weeks old, $n=6$ per group) treated with $250 \mathrm{mg} / \mathrm{kg}$ DCA i.p. daily for 5 days. The data is shown as the mean \pm SEM. Panel A shows glutamate-cysteine ligase activity. The significant difference shown (*) is $p<0.01$. Panel $B$ shows glutathione concentration. Panel $C$ shows glutathione transferase activity with CDNB as a substrate. 

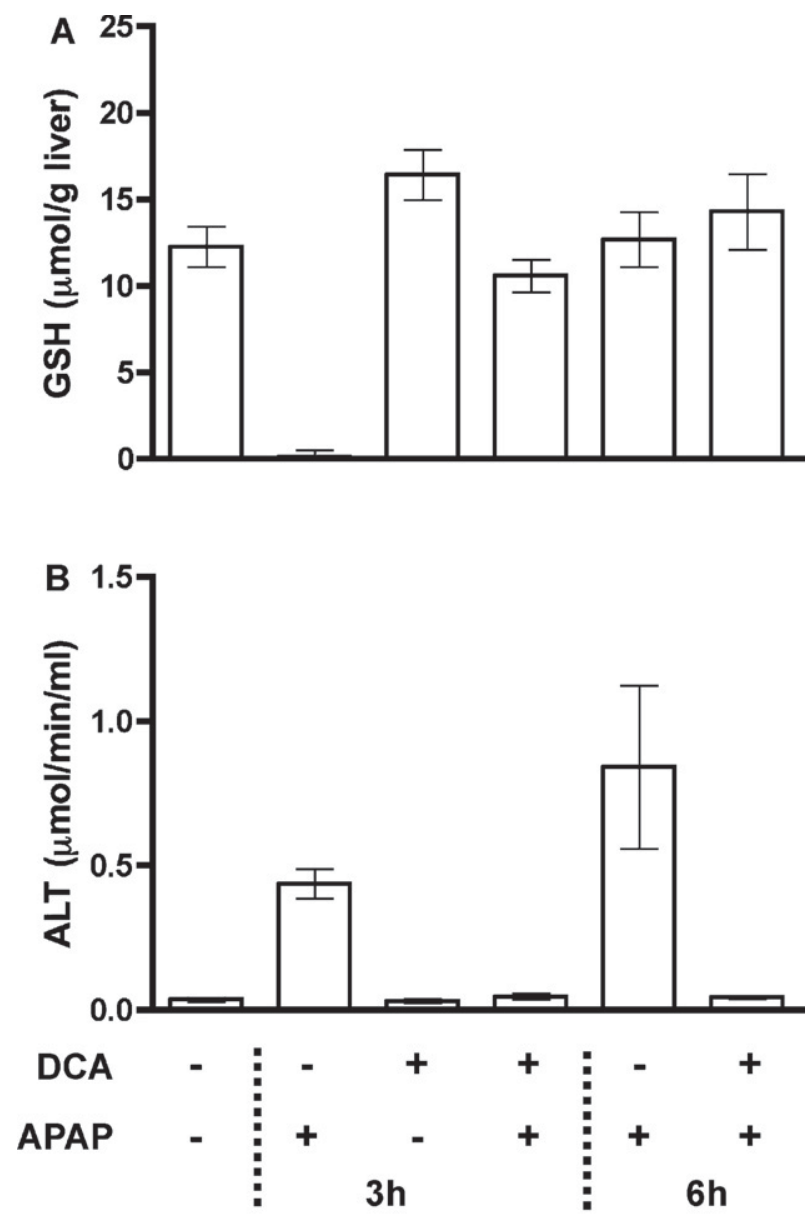

Time after APAP administration

Fig. 3. DCA protects mice from APAP-induced GSH depletion and liver hepatotoxicity. Groups of male BALB/c mice (10-15 weeks old, $n=5-6$ per group) were either untreated or treated daily with $250 \mathrm{mg} / \mathrm{kg}$ DCA i.p. for 5 days. After 5 days, some of these groups received a single i.p. injection of $300 \mathrm{mg} / \mathrm{kg}$ APAP and were sacrificed either $3 \mathrm{~h}$ or $6 \mathrm{~h}$ after the APAP injection, at which time livers were collected for GSH measurement (panel A) and blood was collected for serum ALT measurement (panel B). Data shown as the mean \pm SEM.

in more severe toxicity than occurred in wild-type mice [Grade 3] (Fig. 4d) and resembled more the changes seen $6 \mathrm{~h}$ after the administration of APAP in the wild-type control mice. The administration of APAP for $6 \mathrm{~h}$ to the $\mathrm{Gstz1}^{-1-}$ mice showed similar or greater hepatocyte necrosis than occurred at $3 \mathrm{~h}$.

\subsection{The time course of DCA mediated protection against APAP hepatotoxicity}

Since the protective effect of DCA is closely correlated with the levels of hepatic GSH, we measured liver GSH concentrations $3 \mathrm{~h}$ after the injection of APAP in mice that had been administered daily doses of DCA from 5 days prior, and 1 day prior to the APAP injection and $1 \mathrm{~h}$ after the APAP injection. As shown in Fig. 5, mice receiving DCA up to 1 day before the APAP challenge maintained their hepatic GSH levels. In contrast, mice receiving DCA $1 \mathrm{~h}$ after the APAP injection were not protected and showed significantly diminished hepatic GSH levels. This time course suggests that the DCA mediated protection against APAP hepatotoxicity requires a change in gene expression and is consistent with increased expression of GCLC after DCA treatment (Fig. 1).

As a GST enzyme is thought to be at least partially responsible for the conjugation of NAPQI to GSH and since several GSTs are elevated in Gstz1/- mice [12] we investigated the level of GST activity in the liver. DCA treatment failed to increase total hepatic glutathione transferase activity as measured with 1-chloro-2,4dinitro benzene (CDNB) as a substrate (Fig. 2c). CDNB is a general GST substrate for members of the Alpha, Mu and Pi classes and the data suggests that none of these GSTs are substantially induced or depleted by DCA. Consequently, if any of these GSTs are responsible for the conjugation of NAPQI the reaction must be limited by the availability of GSH.

While the protection mediated by DCA is consistent with an increased capacity for GSH production via augmented GCLC levels after GSH depletion, it is possible that DCA may also activate other protective mechanisms that could prevent the formation of NAPQI or promote its disposition via other pathways. APAP undergoes three primary biotransformation reactions: sulfation, which operates predominantly at low doses, glucuronidation, which is the predominant pathway for APAP elimination at high doses, [22], and a cytochrome P-450-catalysed formation of the toxic electrophilic product, NAPQI, which is eliminated via GSH conjugation [23]. Cyp2E1 is considered to be the major enzyme contributing to NAPQI formation [24]. Real time RT-PCR studies found the level of liver Cyp2E1 mRNA to be unchanged after treatment with DCA for 5 days (data not shown). The expression of sulfotransferase 1A1 (SULT1A1), a major APAP sulfator, was similarly unchanged by DCA (data not shown). UGT activity was assayed in liver microsome extracts and was down-regulated after 5 days of DCA treatment (percent substrate consumed: control $71 \pm 2.8$; DCA-treated $-58.2 \pm 3.0(p=0.011, n=6$ per group $)$ ). Since DCA did not enhance other APAP detoxification pathways or attenuate the pathway that produces NAPQI, it is likely that the rapid replenishment of GSH after its depletion by APAP is the prime mediator of protection.

\section{Discussion}

There has been increasing interest in the pharmacologic effects of DCA since the discovery of its anti-cancer properties in vitro and in vivo and its use in other conditions such as lactic acidosis and inherited mitochondrial diseases [1-4,6,25]. Several clinical trials evaluating the utility of DCA for a number of conditions are currently underway (www.clinicaltrials.gov). Publicity surrounding the potential of DCA as an anticancer agent and its ready availability has generated extensive self medication in patient groups (www.dcasite.com) [7]. Therefore, because of the increased use of DCA in the clinic and the unsupervised self-medication with DCA, it is likely that it will be administered in combination with other drugs. It is therefore important to better understand the toxicology of DCA, especially how well it is tolerated and, importantly, whether any adverse effects result from interactions with other drugs.

DCA is a mechanism-based inhibitor of GSTZ1-1 [8] and the treatment of rodents with DCA results in greatly lowered hepatic GSTZ1-1 activity $[17,26]$. It has been demonstrated that mice with a genetic deficiency in GSTZ1-1 exhibit greatly reduced levels of hepatic GSH and greater hepatic levels of several enzymes including NQO1, the catalytic and modifier subunits of glutamate-cysteine ligase (GCL), and several glutathione tranferases (GSTs) including GSTA1/2, GSTM1-1, GSTM5-5 and GSTP1-1 [12]. It was proposed that the increase in expression of these molecules in Gstz1 $1^{-/-}$mice was mediated via activation of antioxidant response elements (ARE) in their gene promoters, as a consequence of the loss of GSH and oxidative stress attributed to the accumulation of the electrophilic species maleylacetoacetate. Since DCA is an irreversible inactivator of GSTZ1-1, we considered it possible that it could induce the same oxidative stress and gene expression changes as those observed in the livers of $G s t z 1^{-1-}$ mice. However, 

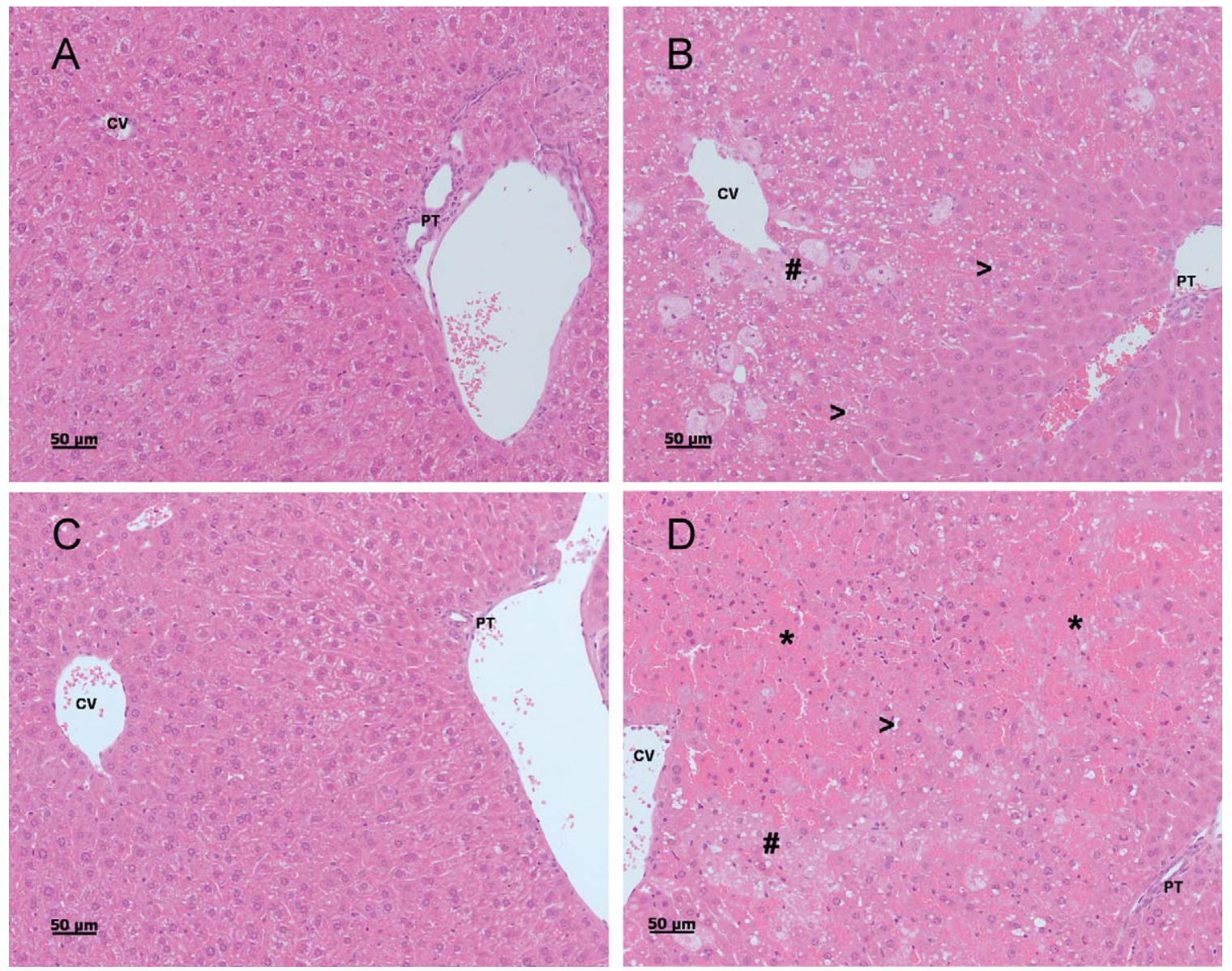

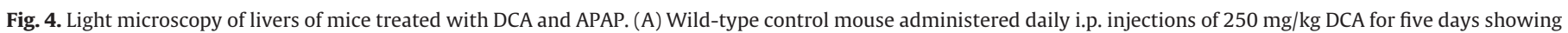

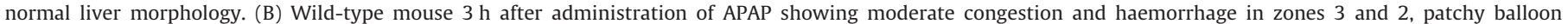

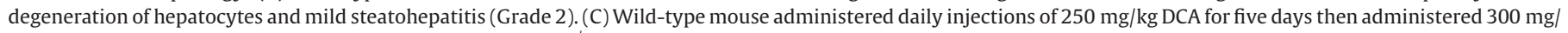

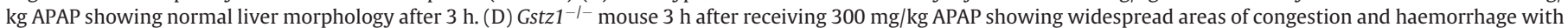

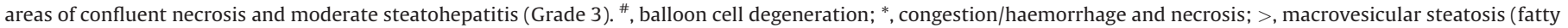
change); CV, central vein; PT, portal tract.

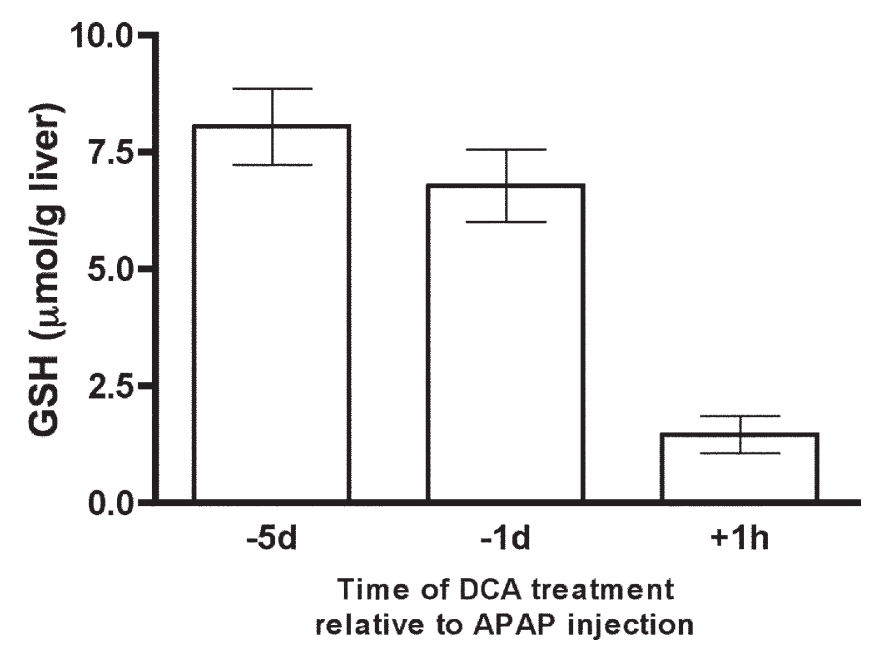

Fig. 5. DCA does not protect from APAP-induced GSH depletion and liver hepatotoxicity if administered after APAP injection. Groups of male BALB/c mice (10-15 weeks old, $n=6$ per group) treated with $250 \mathrm{mg} / \mathrm{kg}$ DCA i.p. daily for 5 days prior to, 1 day prior to, or $1 \mathrm{~h}$ after, a single i.p. injection of $300 \mathrm{mg} / \mathrm{kg}$ APAP. Mice were sacrificed $3 \mathrm{~h}$ after the APAP injection, at which time livers were collected for $\mathrm{GSH}$ measurement. Data shown as the mean \pm SEM. the results obtained in this study indicate that DCA treatment did not completely eliminate hepatic GSTZ1-1 and that GSH levels were elevated rather than depleted. In addition, there was no evidence for the generation of significant oxidative stress and the ARE mediated induction of NQO1 or Alpha, Mu and Pi class GSTs.

Normal therapeutic doses of APAP are primarily metabolised by Phase II glucuronidation and sulfation to non-toxic metabolites [27]. Approximately $5 \%$ is metabolised by the cytochrome P450 system (CYP2E1 in humans) to NAPQI [20]. NAPQI is a toxic metabolite and is presumed to cause the liver toxicity observed upon APAP overdose. Conjugation of GSH to NAPQI is partly nonenzymatic and partly mediated by glutathione transferases [21], and leads to the excretion of NAPQI as a mercapturic acid. Provided there is sufficient GSH present, NAPQI can be readily detoxified and eliminated. In the case of an APAP overdose, GSH is depleted and the build up of NAPQI covalently binds to cellular macromolecules causing hepatotoxicity and cell death $[20,28]$.

We chose a mouse model of APAP overdose in order to determine whether DCA could exacerbate the pathology induced by APAP, since it is well established that APAP toxicity ensues upon GSH depletion, and Gstz1 $1^{-/-}$mice have low hepatic GSH levels. We initially predicted that DCA would sensitize mice to APAPmediated hepatotoxicity since it depletes GSTZ1-1 levels but we made the unexpected discovery that DCA is highly protective. Although total glutathione levels appear to be maintained at 
normal or slightly higher levels after DCA treatment, the key to the protective action of DCA is most likely due to an increased capacity to maintain hepatic GSH levels in response to increased GSH consumption. The synthesis of NAPQI-GSH conjugates normally results in a severe loss of GSH from hepatocytes. If GSH levels are maintained it is likely to be the result of increased de novo synthesis. Mice maintained normal hepatic GSH after APAP challenge only when given DCA at least 1 day in advance of APAP, and DCA did not prevent GSH loss when given $1 \mathrm{~h}$ after APAP (Fig. 5). This time lag between DCA administration and when the hepato-protective effect is manifested suggests that the protective effect requires a gene-induction phase in order express protein(s) that confer protection, presumably GCLC. This delayed protection also precludes DCA as an antidote for APAP overdose.

Glutamate-cysteine ligase (GCL) catalyses the formation of $\gamma$ glutamylcysteine, the first step in the synthesis of GSH. GCL is a heterodimer composed of catalytic (GCLC) and modifier (GCLM) subunits $[29,30]$. In the present study we found increased levels of hepatic GCLC protein and GCL activity in mice treated with DCA for 5 days. However, glutathione synthetase levels appeared to be unchanged. GCL is considered to be the rate-limiting step in glutathione synthesis and is the critical enzyme that dictates the rate of GSH production [30]. The levels of GSH synthesis are normally regulated by the feedback inhibition of GCL by GSH [30]. When GSH is depleted by conjugation to NAPQI, GCL would be released from inhibition to allow synthesis of new GSH. In the present case the higher than normal levels of GCLC induced by DCA would be expected to allow the rapid de novo synthesis of GSH. This result is consistent with a previous study that reported that transgenic mice over-expressing GCL are resistant to acetaminophen-induced hepatotoxicity, but do not exhibit elevated hepatic GSH levels prior to challenge [31].

The mechanism by which DCA causes the up regulation of GCLC expression is not clear and requires further investigation. The timing of the response to DCA and the increase in GCLC protein levels suggests that the response is due to an increase in transcription rather than post-translational modifications. Many previous studies have shown that transcription of GCLC can be up regulated in response to oxidative stress via an ARE and the Nrf2Keap1 pathway (reviewed in [13,32]). However, oxidative stress does not seem to be a major factor here since NQO1, which is also regulated by oxidative stress through an ARE, is only marginally induced in DCA treated mice. It is therefore more likely that the upregulation of GCLC by DCA is mediated by a ROS-independent mechanism.

In this study, our aim was to examine the effects of GSTZ1-1 deficiency mediated by the administration of DCA. This study and our previous work [17] clearly demonstrate that treatment of mice with DCA does not mimic the oxidative stress and other deleterious effects that characterize genetic deficiency of GSTZ1-1. Our discovery that DCA can increase the liver's capacity for GSH synthesis suggests that it may be beneficial in settings where the maintenance of protective levels of GSH are required [33-36], but it may also increase the liver's capacity to detoxify drugs and reduce their effective dose. This produces a dilema for the potential use of DCA in cancer therapy. The GCLC-inducing property of DCA could be exploited in doxorubicin treatment, for example, where it has been demonstrated that cardiac myocyte death, the major limitation of anthracycline treatment, is mediated via reactive oxygen species (ROS) while its anti-cancer properties are ROSindependent [37]. However if this mechanism is happening in tumor cells DCA could lower the efficacy of drugs that rely on oxidative stress for cytotoxicity or where the depletion of glutathione enhances their cytotoxicity [38-40].

In human subjects, as much as $80 \mathrm{mg} / \mathrm{kg}$ i.v. has been used to reduce metabolic acidosis during liver transplantation [41], indicating that doses of the order of those used in the present study are clinically relevant. Further studies in the in vivo setting are necessary to determine whether the overall outcome of hepatic changes vs tumor changes from DCA treatment are a benefit or detriment to specific cancer treatment regimes.

\section{Acknowledgements}

We thank Professor Klaus Matthaei for his original assistance in generating the Gstz1-1- mice. We also acknowledge financial support from NHMRC grant 418071.

\section{References}

[1] Bonnet S, Archer SL, Allalunis-Turner J, Haromy A, Beaulieu C, Thompson R, et al. A mitochondria-K+ channel axis is suppressed in cancer and its normalization promotes apoptosis and inhibits cancer growth. Cancer Cell 2007;11: 37-51.

[2] Michelakis ED, Sutendra G, Dromparis P, Webster L, Haromy A, Niven E, et al. Metabolic modulation of glioblastoma with dichloroacetate. Sci Transl Med 2010;2:31ra4

[3] Sun RC, Fadia M, Dahlstrom JE, Parish CR, Board PG, Blackburn AC. Reversal of the glycolytic phenotype by dichloroacetate inhibits metastatic breast cancer cell growth in vitro and in vivo. Breast Cancer Res Treat 2010;120:253-60.

[4] Stacpoole PW, Gilbert LR, Neiberger RE, Carney PR, Valenstein E, Theriaque DW, et al. Evaluation of long-term treatment of children with congenital lactic acidosis with dichloroacetate. Pediatrics 2008;121:e1223-8.

[5] Chen Y, Cairns R, Papandreou I, Koong A, Denko NC. Oxygen consumption can regulate the growth of tumors, a new perspective on the Warburg effect. PLoS One 2009; 4:e7033.

[6] Stacpoole PW, Henderson GN, Yan Z, James MO. Clinical pharmacology and toxicology of dichloroacetate. Environ Health Perspect 1998;106(Suppl. 4): 989-94.

[7] Pearson H. Cancer patients opt for unapproved drug. Nature 2007;446:474-5.

[8] Tzeng HF, Blackburn AC, Board PG, Anders MW. Polymorphism- and speciesdependent inactivation of glutathione transferase zeta by dichloroacetate. Chem Res Toxicol 2000;13:231-6.

[9] Cornett R, James MO, Henderson GN, Cheung J, Shroads AL, Stacpoole PW. Inhibition of glutathione S-transferase zeta and tyrosine metabolism by dichloroacetate: a potential unifying mechanism for its altered biotransformation and toxicity. Biochem Biophys Res Commun 1999;262:752-6.

[10] Lantum HB, Cornejo J, Pierce RH, Anders MW. Perturbation of maleylacetoacetic acid metabolism in rats with dichloroacetic acid-induced glutathione transferase zeta deficiency. Toxicol Sci 2003;74:192-202.

[11] Lim CE, Matthaei KI, Blackburn AC, Davis RP, Dahlstrom JE, Koina ME, et al. Mice deficient in glutathione transferase zeta/maleylacetoacetate isomerase exhibit a range of pathological changes and elevated expression of alpha, mu, and pi class glutathione transferases. Am J Pathol 2004;165:679-93.

[12] Blackburn AC, Matthaei KI, Lim C, Taylor MC, Cappello JY, Hayes JD, et al. Deficiency of glutathione transferase zeta causes oxidative stress and activation of antioxidant response pathways. Mol Pharmacol 2006;69:650-7.

[13] Forman HJ, Zhang H, Rinna A. Glutathione: overview of its protective roles, measurement, and biosynthesis. Mol Aspects Med 2009;30:1-12.

[14] Baker MA, Cerniglia GJ, Zaman A. Microtiter plate assay for the measurement of glutathione and glutathione disulfide in large numbers of biological samples. Anal Biochem 1990;190:360-5.

[15] White CC, Viernes H, Krejsa CM, Botta D, Kavanagh TJ. Fluorescence-based microtiter plate assay for glutamate-cysteine ligase activity. Anal Biochem 2003;318:175-80.

[16] Blazka ME, Elwell MR, Holladay SD, Wilson RE, Luster MI. Histopathology of acetaminophen-induced liver changes: role of interleukin 1 alpha and tumor necrosis factor alpha. Toxicol Pathol 1996;24:181-9.

[17] Theodoratos A, Tu WJ, Cappello J, Blackburn AC, Matthaei K, Board PG. Phenylalanine-induced leucopenia in genetic and dichloroacetic acid generated deficiency of glutathione transferase Zeta. Biochem Pharmacol 2009;77: 1358-63.

[18] Thompson JA, Franklin CC. Enhanced glutathione biosynthetic capacity promotes resistance to As3+-induced apoptosis. Toxicol Lett 2009;193:33-40.

[19] Cohen SD, Hoivik DJ, Khairallah EA. Acetaminophen-induced hepatotoxicity. In: Plaa GL, Hewitt WR, editors. Toxicology of the liver. Washington, DC: Taylor \& Francis; 1998. p. 159-86.

[20] Hinson JA, Roberts DW, James LP. Mechanisms of acetaminophen-induced liver necrosis. Handb Exp Pharmacol 2010;369-405.

[21] Henderson CJ, Wolf CR, Kitteringham N, Powell H, Otto D, Park BK. Increased resistance to acetaminophen hepatotoxicity in mice lacking glutathione Stransferase Pi. Proc Natl Acad Sci U S A 2000;97:12741.

[22] Hjelle JJ, Klaassen CD. Glucuronidation and biliary excretion of acetaminophen in rats. J Pharmacol Exp Ther 1984;228:407-13.

[23] Dahlin DC, Miwa GT, Lu AY, Nelson SD. N-acetyl-p-benzoquinone imine: a cytochrome P-450-mediated oxidation product of acetaminophen. Proc Natl Acad Sci U S A 1984;81:1327-31. 
[24] Lee SS, Buters JT, Pineau T, Fernandez-Salguero P, Gonzalez FJ. Role of CYP2E1 in the hepatotoxicity of acetaminophen. J Biol Chem 1996;271:12063-7.

[25] Michelakis ED, Webster L, Mackey JR. Dichloroacetate (DCA) as a potential metabolic-targeting therapy for cancer. Br J Cancer 2008;99:989-94.

[26] Anderson WB, Board PG, Gargano B, Anders MW. Inactivation of glutathione transferase zeta by dichloroacetic acid and other fluorine-lacking alphahaloalkanoic acids. Chem Res Toxicol 1999;12:1144-9.

[27] Prescott LF. Paracetamol (acetaminophen): a critical bibliographic review. London: Taylor \& Francis; 1996.

[28] Nelson SD. Mechanisms of the formation and disposition of reactive metabolites that can cause acute liver injury. Drug Metab Rev 1995;27: 147-77.

[29] Meister A, Anderson ME. Glutathione. Annu Rev Biochem 1983:52:711-60.

[30] Richman PG, Meister A. Regulation of gamma-glutamyl-cysteine synthetase by nonallosteric feedback inhibition by glutathione. J Biol Chem 1975;250: 1422-6.

[31] Botta D, Shi S, White CC, Dabrowski MJ, Keener CL, Srinouanprachanh SL, et al Acetaminophen-induced liver injury is attenuated in male glutamate-cysteine ligase transgenic mice. J Biol Chem 2006;281:28865-7.

[32] Lu SC. Regulation of glutathione synthesis. Mol Aspects Med 2009;30: 42-59.

[33] Bounous G. Whey protein concentrate (WPC) and glutathione modulation in cancer treatment. Anticancer Res 2000;20:4785-92.
[34] Sian J, Dexter DT, Lees AJ, Daniel S, Agid Y, Javoy-Agid F, et al. Alterations in glutathione levels in Parkinson's disease and other neurodegenerative disorders affecting basal ganglia. Ann Neurol 1994;36:348-55.

[35] Magnani M, Fraternale A, Casabianca A, Schiavano GF, Chiarantini L, Palamara AT, et al. Antiretroviral effect of combined zidovudine and reduced glutathione therapy in murine AIDS. AIDS Res Hum Retroviruses 1997:13:1093-9.

[36] Yamauchi A, Tsuyuki S, Inamoto T, Yamaoka Y. Liver immunity and glutathione. Antioxid Redox Signal 1999;1:245-53.

[37] Wang S, Konorev EA, Kotamraju S, Joseph J, Kalivendi S, Kalyanaraman B. Doxorubicin induces apoptosis in normal and tumor cells via distinctly different mechanisms. intermediacy of $\mathrm{H}(2) \mathrm{O}(2)$ - and p53-dependent pathways. J Biol Chem 2004;279:25535-43.

[38] Zhang K, Chew M, Yang EB, Wong KP, Mack P. Modulation of cisplatin cytotoxicity and cisplatin-induced DNA cross-links in HepG2 cells by regulation of glutathione-related mechanisms. Mol Pharmacol 2001;59:837-43.

[39] Brozovic A, Ambriovic-Ristov A, Osmak M. The relationship between cisplatininduced reactive oxygen species, glutathione, and BCL-2 and resistance to cisplatin. Crit Rev Toxicol 2010;40:347-59.

[40] Estrela JM, Ortega A, Obrador E. Glutathione in cancer biology and therapy. Crit Rev Clin Lab Sci 2006;43:143-81.

[41] Shangraw RE, Lohan-Mannion D, Hayes A, Moriarty RM, Fu R, Robinson ST. Dichloroacetate stabilizes the intraoperative acid-base balance during liver transplantation. Liver Transpl 2008;14:989-98. 